\title{
Examination of Myxobacteria by Scanning Electron Microscopy
}

\author{
K. E. MCNEIL and V.B. D. SKERMAN \\ Department of Microbiology, University of Queensland, Brisbane, Australia
}

\begin{abstract}
Fruiting bodies of representative genera of the order Myxobacterales Jahn 1915 were examined by using a "Stereoscan" electron microscope (Cambridge Instrument Co.). Species of Myxococcus, Chondrococcus, Melittangium, Chondromyces, Archangium, and Stigmatella were examined. The instrument was found to be of value in studying the detail of fruiting bodies.
\end{abstract}

Myxobacteria undergo a life cycle involving the cellular aggregation of vegetative rods into a fruiting body. The rods within the fruiting body form resting cells known as myxospores. The fruiting bodies of certain species have been examined by transmission electron microscopy $(1,3,7)$.

The scanning electron microscope (SEM) gives considerable depth of focus at high magnification, an advantage over the light microscope. It was therefore thought useful to examine the morphology of fruiting bodies of myxobacteria with the SEM.

\section{MATERIALS AND METHODS}

Strains studied. Myxobacteria were isolated from soil and bark from southeast Queensland by using the methods of Singh (5) and Peterson (4). The isolates from soil were identified as Chondromyces apiculatus MQ47, Myxococcus virescens MQ4, Myxococcus fulvus MQ41, Chondrococcus coralloides MQ25, Chondromyces crocatus MQ101, and Stigmatella erecta MQ42. The isolates from bark were identified as Stigmatella aurantiaca MO89, Melittangium lichenicolum MQ21, and Archangium primigeninm MQ19. The MQ numbers are the accession numbers allocated to the cultures in the collection of the Department of Microbiology, University of Queensland. Fruiting bodies of Chondromyces pediculatus and Chondromyces catenulatus were examined but not obtained in pure culture.

Preparation for the SEM. In some instances the specimens were examined without prior fixation. However, to preserve the more delicate fruiting bodies and other fruiting bodies at a certain stage of growth, the specimens were fixed over osmium vapor. For mounting in the SEM, small blocks of agar or pieces of bark, bearing fruiting bodies, were attached to the specimen stub, the face of which was pretreated a few seconds previously with adhesive removed from "Bear" brand (3M) adhesive tape with chloroform. The specimens were coated with gold after evacuation in a Siemens vacuum evaporator.

\section{RESULTS AND DISCUSSION}

The scanning electron micrographs of the fruiting bodies are shown in Fig. 1 to 24 .

A problem was encountered when examining strains of Chondromyces spp. and Stigmatella aurantiaca. The fruiting bodies of these organisms tended to accumulate charge, with the result that compact clusters of cysts repelled each other, causing rapid dismembering of the fruiting bodies while under observation in the SEM. Black lines across the photographs (Fig. 13) resulted from charging. The problem was partly overcome by fixation in osmium vapor. For any particular strain, there was no difference in appearance between the individual cysts from osmium-fixed and unfixed fruiting bodies of the above strains as observed under the SEM.

The process of evacuation prior to gold coating must cause substantial dehydration and may result in folding of the walls of cysts (Fig. 14) and the contraction of slime into an apparent membrane over myxospores in noncyst-forming species (Fig. 20). It is difficult to resolve these folds (if they existed) in the unevacuated specimens with the light microscope.

The distribution of myxospores in the cysts of Melittangium lichenicolum was revealed by the folding of the cyst walls (Fig. 15). Large numbers of myxospores within the cyst of $M$. lichenicolum may physically prevent extensive folding; in the cysts of other species the myxospores may be fewer in number relative to the size of the cyst, and no physical evidence of their presence is revealed in the folding of the cyst walls. Our own observations of thin sections of cysts of species of Stigmatella and Chondromyces, confirming those of McCurdy and Khouw (3) and Voelz and Reichenbach (7), have revealed substantial areas of matrix. When cysts of $M$. lichenicolum are crushed under a 

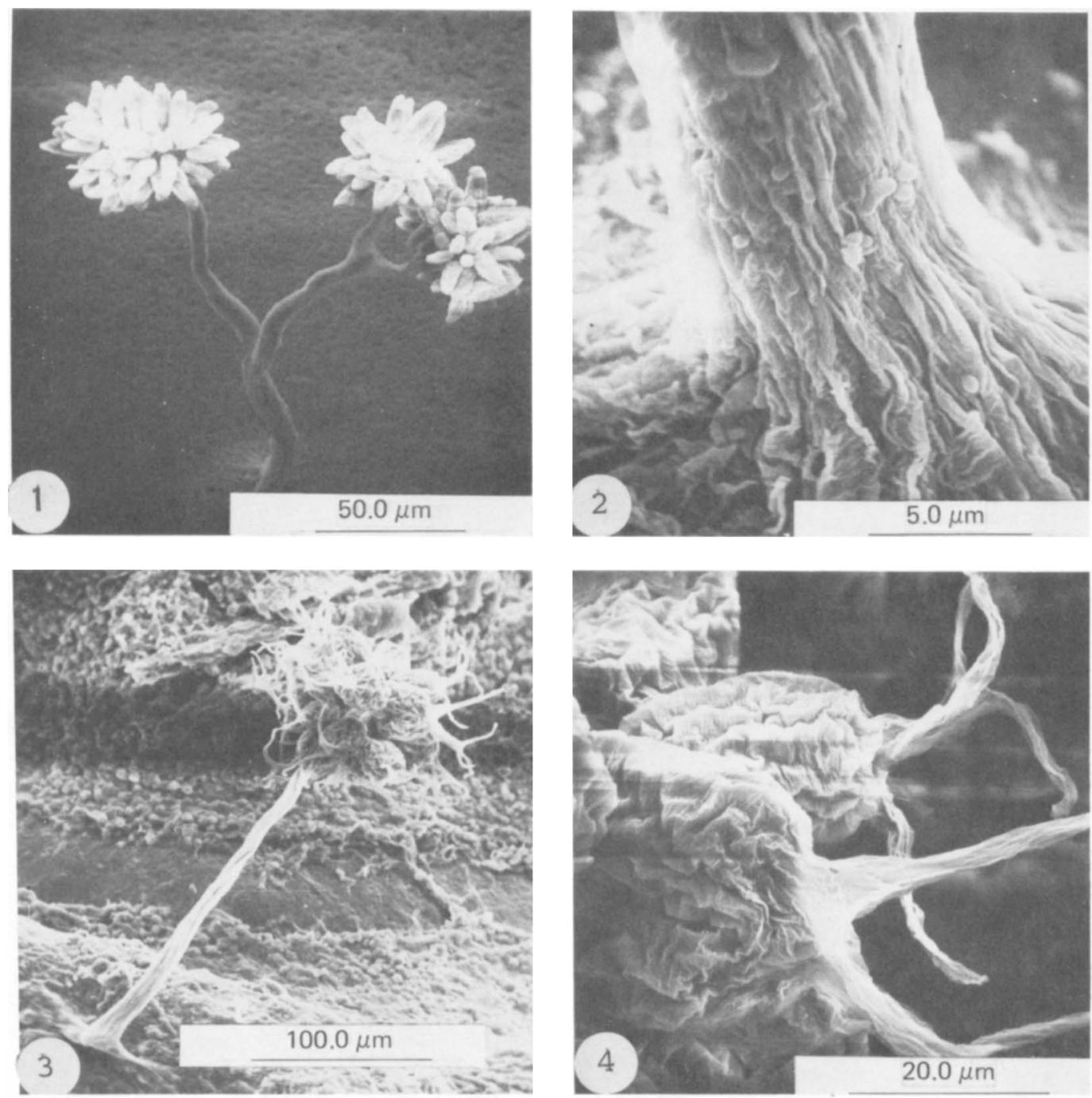

FIG. 1. Chondromyces crocatus MQ101. Fruiting body on agar. $\times 374$. Fixed in osmium vapor.

FIG. 2. Chondromyces crocatus $M Q 101$. Stalk. $\times 4,080$. Fixed in osmium vapor.

FIG. 3. Chondromyces apiculatus MQ47. Fruiting body on agar. $\times 200$. Fixed in osmium vapor.

FIG. 4. Chondromyces apiculatus MQ47. Cysts and slime appendages $\times 1,150$. Fixed in osmium vapor.

cover slip, the myxospores adhere together in sheaves. This is in agreement with the observations by McCurdy (2). Cysts of Chondromyces and Stigmatella readily liberate their myxospores. This suggests a marked variation in the viscosity of the matrix in mature cysts.

The stalk of $S$. aurantiaca (Fig. 13) appears superficially different from those of the four Chondromyces species (Fig. 2, 3, 10, and 11).

A comparison between several strains of Myxococcus fulvus and Myxococcus virescens has revealed a consistent difference in their fruiting bodies. $M$. virescens revealed myxo- spores enveloped in a folded blanket of condensed slime (Fig. 19, 20). In contrast, $M$. fulvus appears to have a uniform surface broken only by depressions, presumably caused by a partial collapse of the myxospores under vacuum (Fig. 21-24).

Fixation of the fruiting bodies in osmium vapor prior to examination did not prevent the formation of these depressions. Thin sections of myxospores (6) revealed no lateral depressions. These depressions are similar to those seen under the SEM by Williams and Davis (8) when examining spores of Streptomyces viridosporus. 

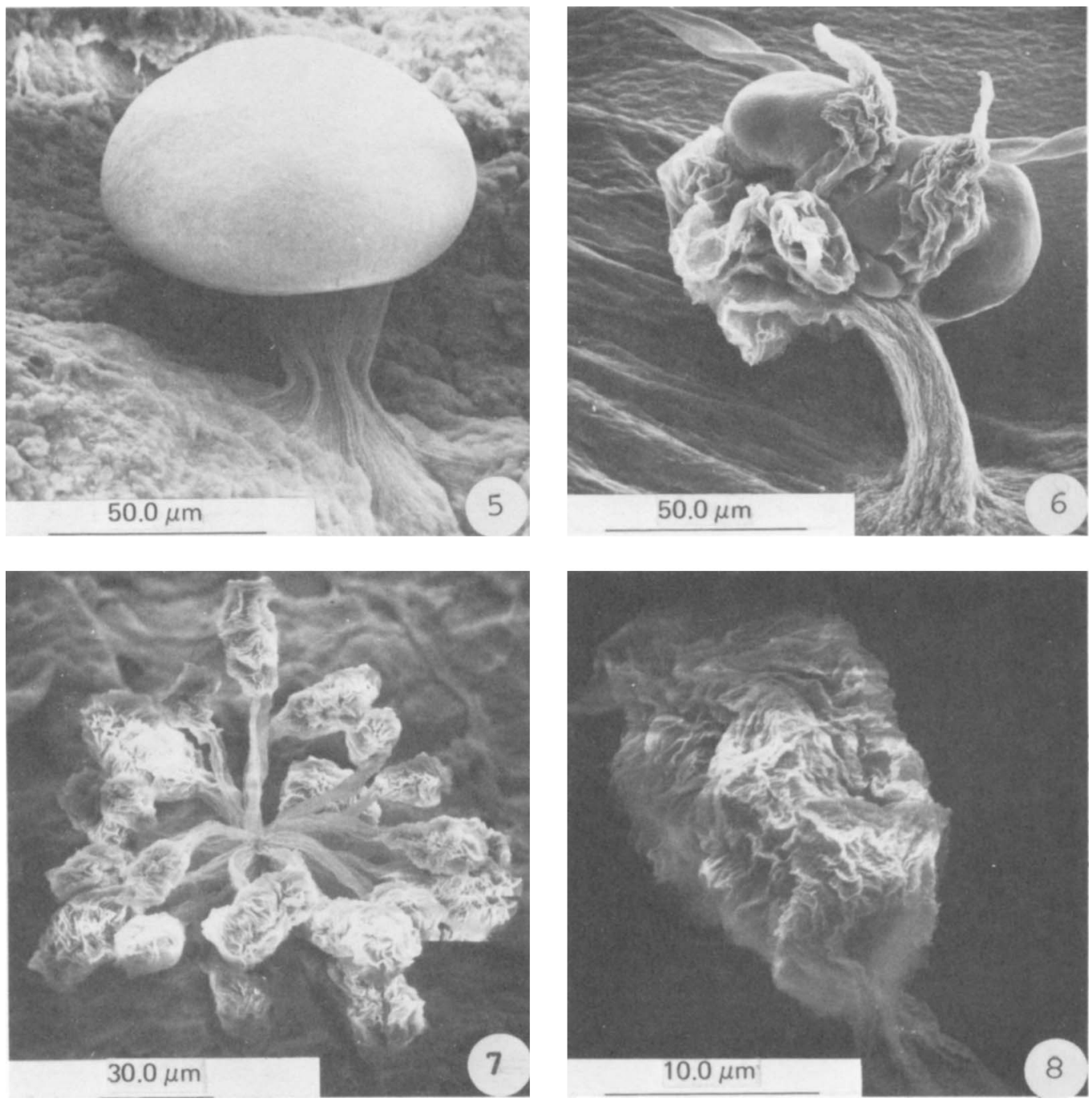

FIG. 5. Chondromyces apiculatus MQ47. Early stage in formation of fruiting body. $\times 488$. Fixed in osmium vapor.

FIG. 6. Chondromyces apiculatus $M Q 47$. Differentiation into cysts. $\times 446$. Fixed in osmium vapor.

FIG. 7. Chondromyces pediculatus. Fruiting body on bark showing cysts attached to stalk by pedicels. $\times 467$. FIG. 8. Chondromyces pediculatus. Cyst and pedicel. $\times 2,465$. 

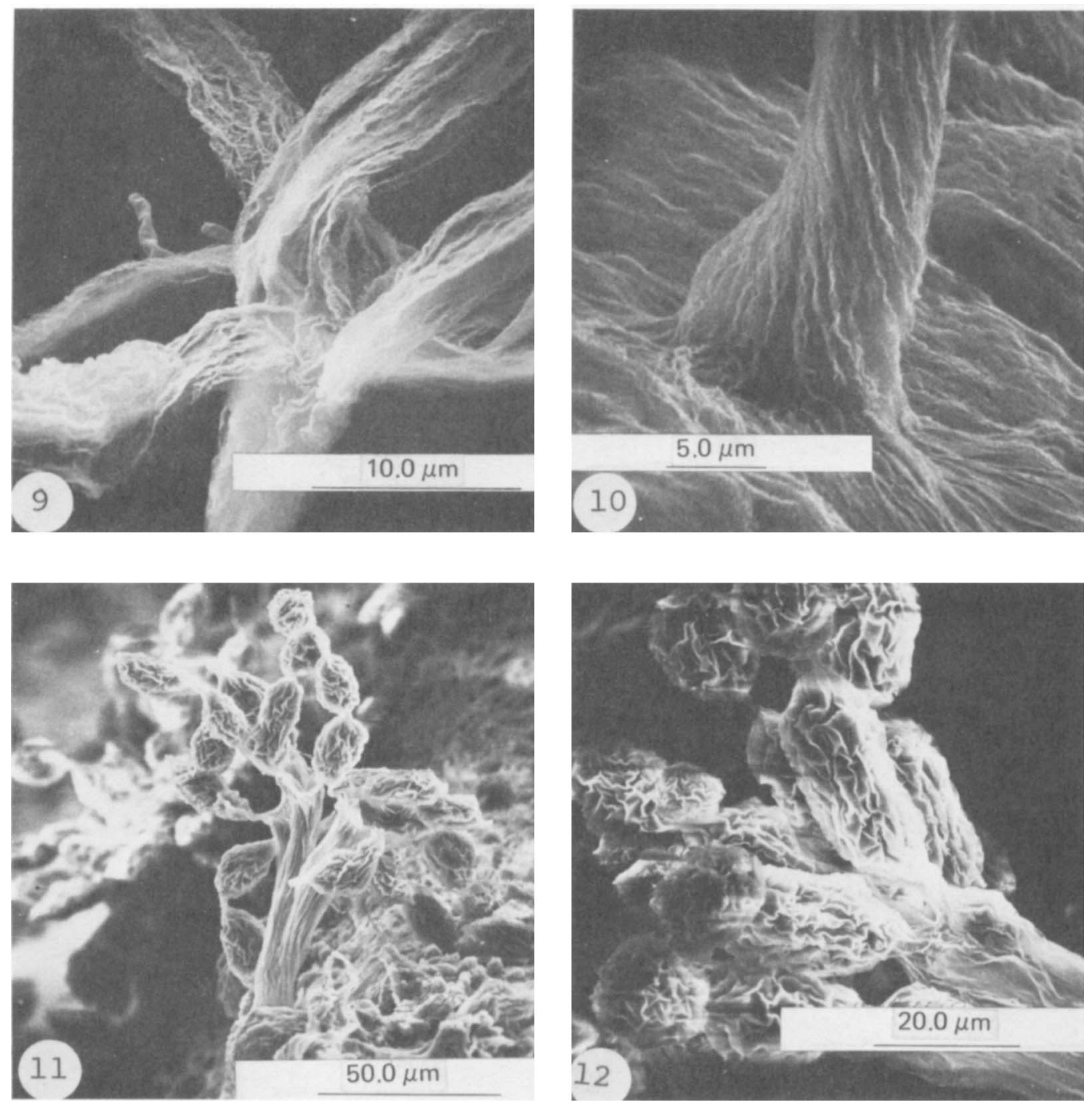

FIG. 9. Chondromyces pediculatus. Junction of stalk and pedicels. $\times 2,465$.

FIG. 10. Chondromyces pediculatus. Base of stalk. $\times 2,900$.

FIG. 11. Chondromyces catenulatus. Fruiting body on bark showing cysts in characteristic chains. $\times 425$. Fixed in osmium vapor.

FIG. 12. Chondromyces catenulatus. The point of division of the main stalk into the cyst chains is shown. $\times 850$. Fixed in osmium vapor. 

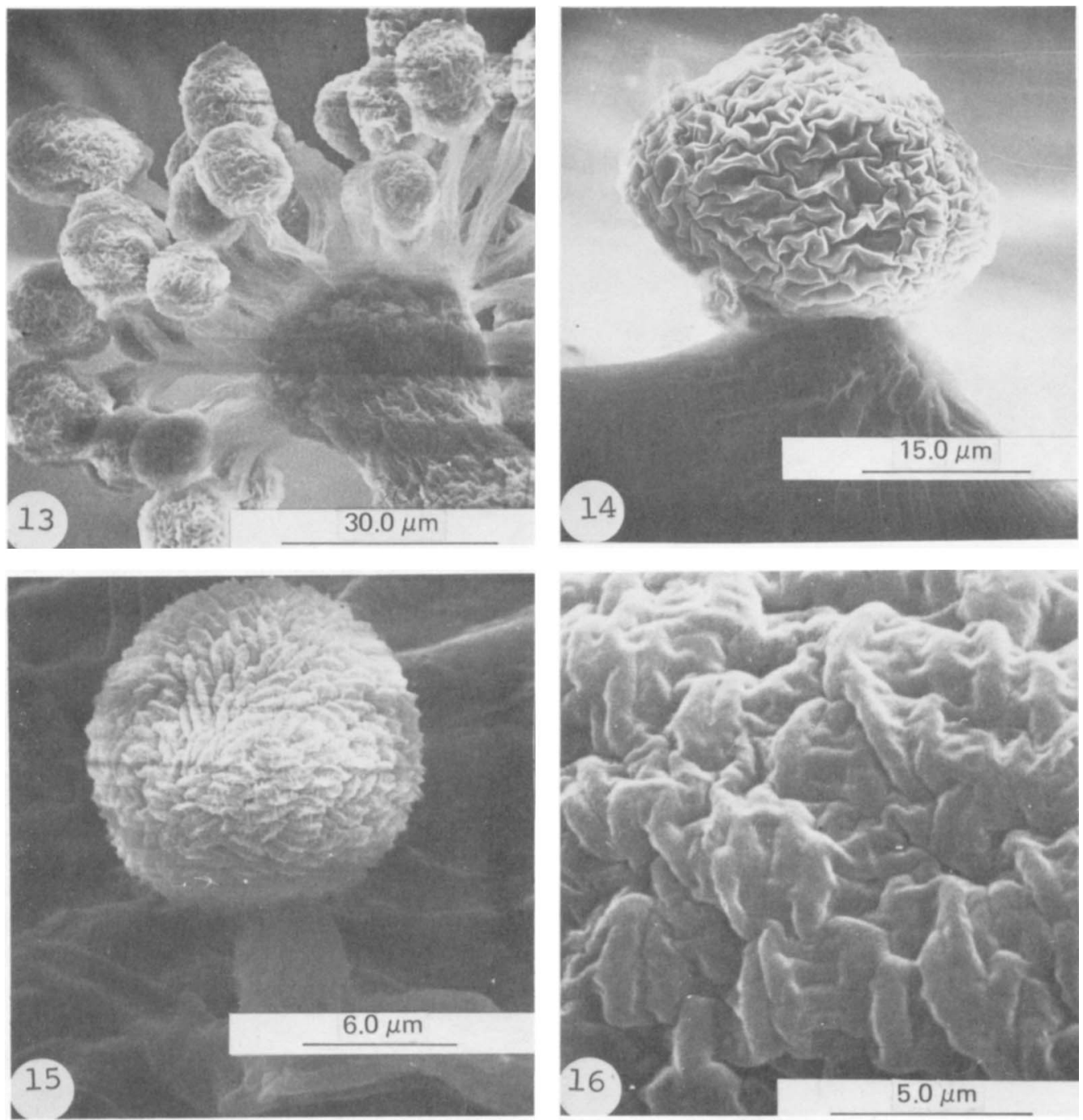

FIG. 13. Stigmatella aurantiaca MQ89. Fruiting body on bark showing cysts attached to stalk by pedicels. $\times 952$. Fixed in osmium vapor.

FIG. 14. Stigmatella erecta MQ42. Fruiting body on agar. $\times 1.215$.

FIG. 15. Melittangium lichenicolum MQ21. The myxospores within the cyst are revealed. $\times 2,890$.

FIG. 16. Archangium primigenium MQ19. Surface of a fruiting body. $\times 5,500$. 

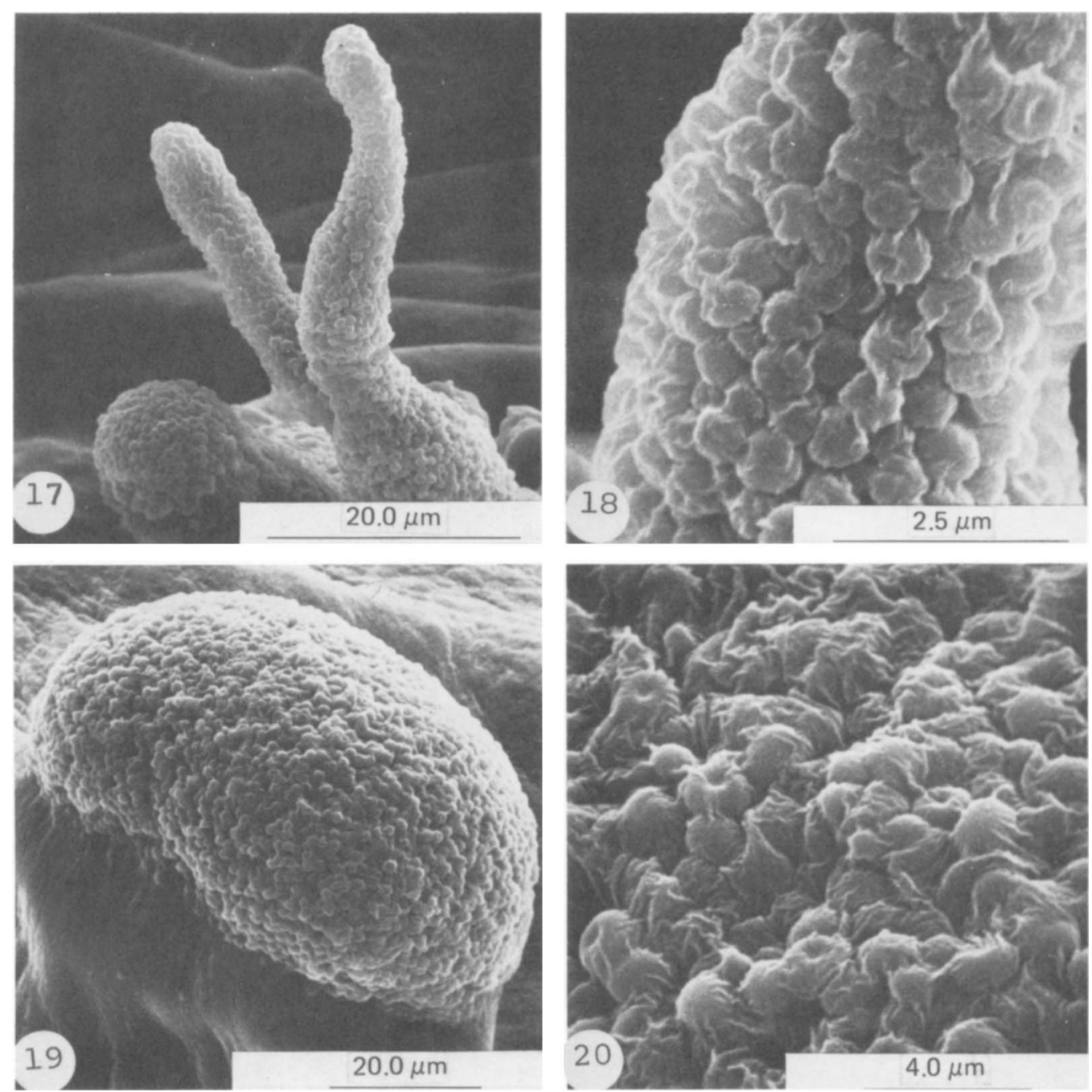

FIG. 17. Chondrococcus coralloides MQ25. Typical fruiting body on agar. $\times 1,530$.

FIG. 18. Chondrococcus coralloides. A slime membrane is visible around the fruiting body. $\times 8,500$.

FIG. 19. Myxococcus virescens MQ4. Fruiting body on agar. $\times 1,000$.

FIG. 20.Myxocotcus virescens MQ4. A-slime membrane is visible. $\times 5,000$. 

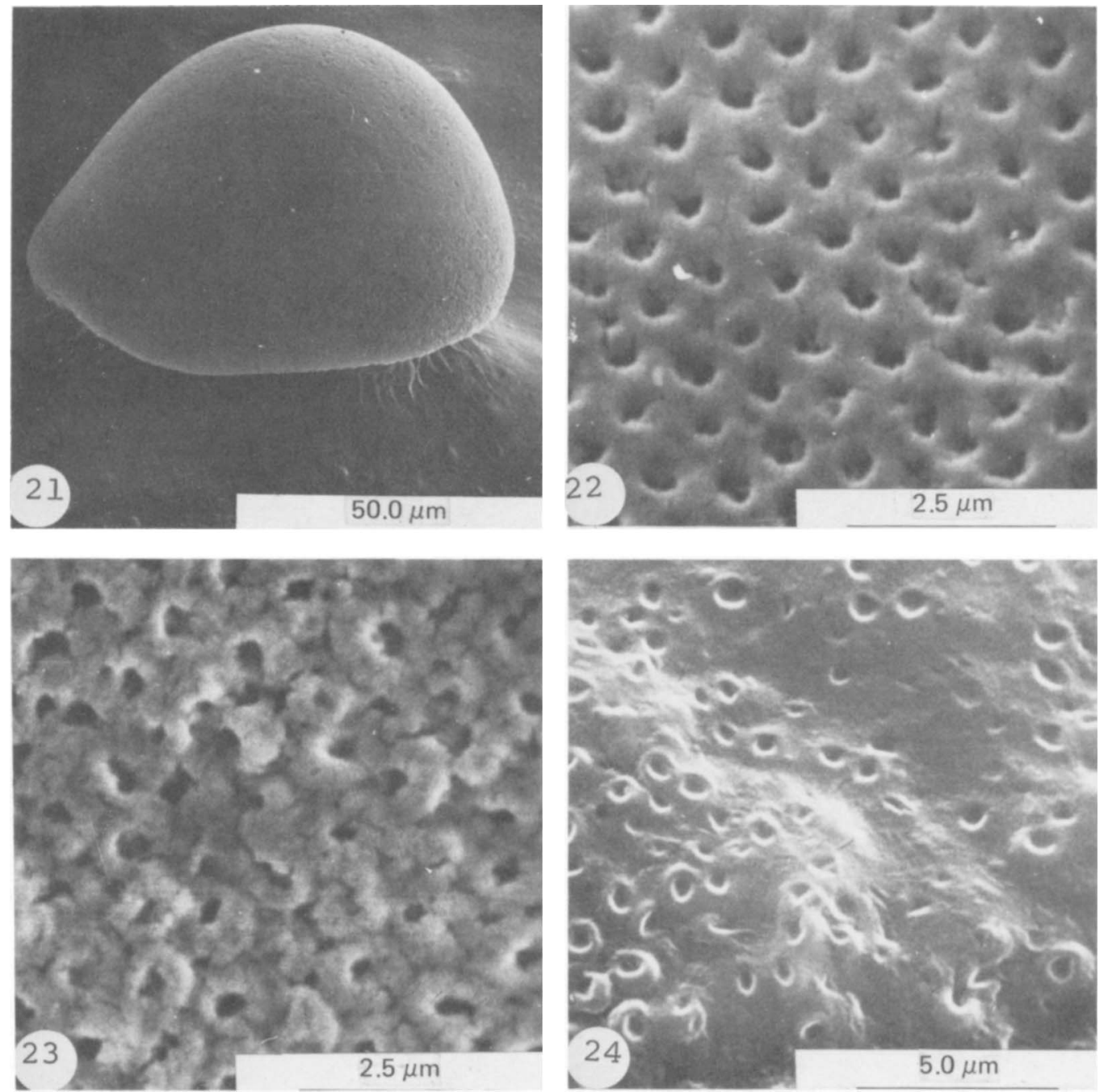

FIG. 21. Myxococcus fulvus MQ41. Typical fruiting body on agar. $\times 573$.

FIG. 22 and 23. Myxococcus fulvus MQ41. Surface detail of two different areas of the fruiting body. $\times 10,000$.

FIG. 24. Myxococcus fulvus $M Q 2$. Surface detail of fruiting body which was different to $M$. fulvus $0041 . \times$ 5,000 . 
The difference observed between $M$. fulvus and $M$. virescens may be due solely to the difference in the ratio of myxospores to slime in the fruiting bodies. The surface appearance of Chondrococcus coralloides was similar to that of $M$. virescens (Fig. 18). Since the differentiation of Chondrococcus from Myxococcus is based solely on deliquescence versus nondeliquescence of the slime of the fruiting body, the continued separation of these two genera appears unjustified.

\section{ACKNOWLEDGMENTS}

We wish to acknowledge the assistance of J. Hardy and R. Grimmer, who operated the electron microscope, and the assistance of Kim Marshall.

\section{LITERATURE CITED}

1. McCurdy, H. 1968. Light and electron microscope studies on the fruiting bodies of Chondromyces crocatus. Arch. Mikrobiol. 65:380-390.

2. McCurdy, H. D. 1971. Studies on the taxonomy of the Myxobacterales. IV. Melittangium. Int. J. Syst. Bacteriol. 21:50-54.

3. McCurdy, H., and B. T. Khouw. 1969. Studies on Stigmatella brunnea. Can. J. Microbiol. 15:731738.

4. Peterson, J. E. 1969. The fruiting myxobacteria: their properties, distribution and isolation. J. Appl. Bacteriol. 32:5-12.

5. Singh, B. N. 1947. Myxobacteria in soils and composts; their distribution, number and lytic action on bacteria. J. Gen. Microbiol. 1:1-10.

6. Voelz, H., and M. Dworkin. 1962. Fine structure of Myxococcus xanthus during morphogenesis. J. Bacteriol. 84:943-952.

7. Voelz, H., and H. Reichenbach. 1969. Fine structure of fruiting bodies of Stigmatella aurantiaca (Myxobacterales). J. Bacteriol. 99:856-866.

8. Williams, S. T., and F. L. Davis. 1967. Use of a scanning electron microscope for the examination of actinomycetes. J. Gen. Microbiol. 48:171-177. 